INPLASY

PROTOCOL

To cite: Xiao et al. Effects of Aromatherapy on Agitation and Aggression in Cognitive Impairment: A Protocol for Meta-Analysis. Inplasy protocol 202160095. doi: 10.37766/inplasy2021.6.0095

Received: 25 June 2021

Published: 25 June 2021

Corresponding author: Shudan Xiao

104754191375@henu.edu.cn

Author Affiliation:

College of nursing and health, Henan University, Kaifeng City, Henan Province, China.

Support: 192102310517;

Wjlx2020449.

Review Stage at time of this submission: Preliminary searches.

Conflicts of interest:

None declared.

\section{Effects of Aromatherapy on Agitation and Aggression in Cognitive Impairment: A Protocol for Meta-Analysis}

Xiao, SD1; Wang, Y2; Duan, SM³; Li, B4 .

Review question / Objective: What are the effects of aromatherapy onagitation and aggression in adults with cognitive impairment or dementia?

Condition being studied: Aging-related cognitive decline have become the leading cause of disabilities among older adults. $\mathrm{MCl}$ is an intermediate stage between normal aging and dementia. Dementia a syndrome in which there is deterioration in memory, thinking, behavior and the ability to perform everyday activities.

Information sources: This meta-analysis was conducted under PRISMA guidelines. Nine electronic literature databases were searched: Web of Science, PubMed, PsycINFO, Embase, Cochrane Library, Chinese Wan-fang database, CNKI, VIP digital database from the inception of the databases up to 27 April 2021.

INPLASY registration number: This protocol was registered with the International Platform of Registered Systematic Review and Meta-Analysis Protocols (INPLASY) on 25 June 2021 and was last updated on 25 June 2021 (registration number INPLASY202160095).

\section{INTRODUCTION}

Review question / Objective: What are the effects of aromatherapy onagitation and aggression in adults with cognitive impairment or dementia?
Condition being studied: Aging-related cognitive decline have become the leading cause of disabilities among older adults. $\mathrm{MCl}$ is an intermediate stage between normal aging and dementia. Dementia a syndrome in which there is deterioration in 
memory, thinking, behavior and the ability to perform everyday activities.

\section{METHODS}

Participant or population: Participants are people (above 55 years old) diagnosed with $\mathrm{MCl}$ or dementia. Probable $\mathrm{MCl}$ or dementia that people do not have a confirmed diagnosis but meeting the criteria of $\mathrm{MCl}$ or dementia (e.g. according to MMSE, MoCA, DRS score) were also included. Studies that included a mixed population of $\mathrm{MCl} / d e m e n t i a$ and healthy participants were excluded if they did not present data separately for $\mathrm{MCl} /$ dementia.

Intervention: Aromatherapy is a form of complementary medicine that uses plant essential oil (EO) to treat health care.

Comparator: Control groups included a passive control group (e.g. health education, usual care, no intervention).

Study designs to be included: Only randomized controlled trials (RCTs) were included.

Eligibility criteria: We selected all publications and screened studies and included them in this systematic review according to PICOS approach (Participants, Intervention, Comparison, Outcome, Study Design): 1. Types of participants: The patients who met the relevant formal diagnoses diagnostic criteria of cognitive impairment in WHO International Classification of diseases (ICD10) (Organization, 1993) and the Diagnostic and Statistical Manual of Mental Disorders, Fourth Edition (DSM-IV) (Md, 1994) or cognitive test scores consistent with cognitive impairment such as Alzheimer's Disease Assessment Scalecognitive subscale (ADAS-Cog) (Rosen et al., 1994) and the Mini-Mental State Examination (MMSE) (Folstein, Folstein, \& McHugh, 1975). 2. Types of interventions: We included trials which used fragrance from plants in an intervention defined as aromatherapy for people with cognitive impairment, and there were no restrictions on setting, frequency, fragrance and duration. 3. Types of comparisons: The control group was placebo aromatherapy, usual care or no treatment. 4. Types of outcomes: The outcomes of interest were any measure of agitation and aggression. 5 . Measurement tools: the Cohen Mansfield Agitation Inventory (CMAI), Pittsburgh Agitation Scale (PAS) and the Neuropsychiatric Inventory (NPI) Agitation/ Aggression domain. 6.Types of study design: peer-reviewed articles with RCT design including an active or passive control group and end date 27 February 2021. Studies without complete information but met our inclusion criteria were included in the narrative review The exclusion criteria included the following: (1) studies that effect sizes without raw data; (2) conference abstracts; (3) case reports or (4) not written in Chinese or English.

Information sources: This meta-analysis was conducted under PRISMA guidelines. Nine electronic literature databases were searched: Web of Science, PubMed, PsycINFO, Embase, Cochrane Library, Chinese Wan-fang database, CNKI, VIP digital database from the inception of the databases up to 27 April 2021.

Main outcome(s): The primary outcomes were agitation and aggression.

Quality assessment / Risk of bias analysis: The Cochrane risk of bias tool was used for quality appraisal. This tool assessed bias from six domains: selection bias, performance bias, detection bias, attrition bias, reporting bias, and other bias.

Strategy of data synthesis: Meta-analysis was conducted using RevMan 5.4 software, and data pooling was performed when at least three studies reporting on the same outcome; otherwise narrative analysis was used. The mean change and standard deviation (SD) were extracted directly or were calculated from the published data. When the studies provided statistics of means, SD, and the number of participants of each group at baseline and postintervention. The mean change was calculated by subtracting the final mean from the baseline mean. The SD of mean 
change was computed with the assumption that the correlation coefficient is $\mathbf{0 . 5}$.

Subgroup analysis: Sub-group analysis was performed based on the diagnosis, age of participants, mode of combination, session length, session frequency, supervision, and intervention setting.

Sensitivity analysis: The sensitivity analysis using stata15.1 software. The sensitive analysis is performed by excluding study one by one. The sensitivity is low and the result are of stability if there are no significant changes that appear in the result before and after the exclusion; if not, it indicates a high sensitivity and unstable result.

Country(ies) involved: China.

Keywords: aromatherapy, cognitive impairment, agitation, aggression, metaanalysis.

Contributions of each author:

Author 1 - Shudan Xiao.

Email: 104754191375@henu.edu.cn

Author 2 - Ying Wang.

Author 3 - Shumin Duan.

Author 4 - Bo Li. 